\title{
Performance Analysis of Continuity Equation and Its Applications
}

\author{
Md. Towhiduzzaman
}

\begin{abstract}
This study has been focused on the Continuity Equation and its applications. A continuity equation illustrates the conveyances of some quantity. Principally, it is uncomplicated as well as powerful when applied to a conserved quantity. Contrarily, it can be generalized to apply to any extensive capacity. In this respect, firstly, it has been analyzed on the Basic Hydrodynamics and the Continuity Equations. It has also been demonstrated on the applications of Continuity Equation in which one can find out the different continuities for different areas of taps at different heights. Besides, there is a comparison also on the Theoretical Flow Rate and Experimental Flow Rate of fluid under the sluice gate by using Continuity Equation and Bernoulli Equation. So, it can be experimented here on the Theoretical Flow Rate and Experimental Flow Rate to make a good agreement on the subject matters.
\end{abstract}

Index Terms - Continuity equation, fluids, flow rate, incompressible flow.

\section{INTRODUCTION}

The Continuity Equation expresses the rate of production of mass within a projected dimension that is equal to the net outflow of mass from the volume. It can be said that the matter is neither being created nor destroyed. We may establish a Continuity Equation in fluid dynamics is a differential equation that illustrates the transport of some sort of conserved quantities. A vast variety of physics can be explained with continuity equations where mass, energy, momentum, electric charge and other natural quantities are conserved. A basic principle of science and engineering is the conservation of mass. So, the continuity equation is an outcome of those basic principles in a particularly convenient form of the analysis of materials processing of operations [1]-[2].

We may set up a new flow as a traffic flow. The Traffic flows in any rate of speed limit denote the mobility between lanes. The continuity equation, relating to the traffic flow, articulates the relationship among the density, flow and speed. The density marginalizes the flow divided by space and speed. The formulation of this equation has two significant hypotheses. One is that the spacing of speed which is constant and it means the un-congested culminations associated with moderation to slightly high volumes. The postulation is that the homogeneous traffic overcomes on the vehicle works that are identical and behavioral within the authoritarian lane disciplines. If the

Published on November 27, 2016.

Md. Towhiduzzaman is a Lecturer in Mathematics, Department of Electrical \& Electronic Engineering, Uttara University (UU), Dhaka-1230, Bangladesh. (Email: towhid.math.iu@gmail.com) continuity equation is applicable over non-homogeneous transferred conditions, one can carry out a research program concerning data collection of density, flow, and speed which is essential for diverged three mid-blocks. This process of data compilation took place when some un-congested situations are prevailed with the moderation to slightly high volumes. Relating the regular density, obtained from experiential densities in the field, reveals whether the continuity equation exactly calculates the average density under non-homogeneous traffic conditions or not [3]-[4].

A variety of scholars explain this continuity equation in several ways. In 1903, Resc elucidated the convergence explanations of the channel flow as well as he calculated the figure of the total errors in the continuity equation for the whole spheres. Plus, Schrödinger published his lines of investigation in 1926. He also narrated down the complex or conjugated equations and confirmed that the taking of them together was not difficult to deduce for a continuity equation [5]-[6].

The continuity equation carries on the preservation of mass/charge/ probability of some closed systems. This equation relates the spatial distribution of the flux compactness corresponding to the sequential variations of the particle density (charge/mass). Normally, this equation is obtained from the equation of motion. The motion of any unstopped charge/mass allocation can be idealized as a continuum (field or fluid). It guarantees that there is no thrashing or whipping of such quantities. This equation renders us with the statements about the system. The message is carried from one point to another by an elementary field wave. We, consequently, figure out that this sort of equation should reveal this property. For electromagnetic field, the basics about the motion of the charges are synchronized by photons since the electrons have particle-wave temperaments. The current and densities of charge/mass are harmonized if we formulate our fundamental fractional and deferential equations using with ions. Thus, during having a partial deferential equation, our advancement is algebraically-based. Besides, generating the recognized continuity equation, this theory is moving towards to lead any new equations [7]-[8].

\section{The ConTINUITY EQUATION}

The act of Conservation of Mass states that mass can be neither created nor ruined. Fixed flow process, run where the flow rate needs not change over time through a optimal volume where the accumulated mass in the control volume has no change to the implements that inflow equal's outflow. This phenomenon is signified as the Equation of 
Continuity. The Continuity Equations bear the local form of conservational decrees. The entire Continuity Equations articulate the identical thoughts roughly to intensify the totality of the conserved proximity inside any region that can only be changed by the quantity going through or out of the region of the frontiers. A preserved mass cannot be increased or decreased until it is only moveable from place to place.

Thinking in the motionless and differential voluminous elements of length $\Delta x$, a width $\Delta y$ and height $\Delta z$ in the form of a Cartesian organizing system, it has been illustrated in Fig. 1.

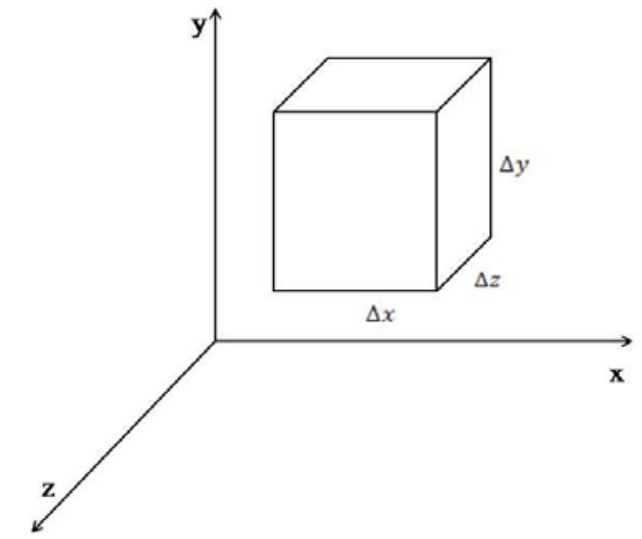

Fig. 1. A randomization for the differential and dimensional components.

The maintenance of mass for these voluminous components $(\Delta V=\Delta x \Delta y \Delta z$ ) could be expounded vocally as:

Velocity of altering of the mass in $\Delta V$

$=$ Velocity of the mass transported into $\Delta V$ - Velocity of mass transported out of $\Delta V$.

Articulation of mathematics is like this:

$$
\begin{aligned}
\Delta x \Delta y \Delta z \frac{\partial \rho}{\partial t}=\Delta & y \Delta\left[\left.\left(\rho v_{x}\right)\right|_{x}-\left.\left(\rho v_{x}\right)\right|_{x+\Delta x}\right] \\
& +\Delta x \Delta z\left[\left.\left(\rho v_{y}\right)\right|_{y}-\left.\left(\rho v_{y}\right)\right|_{y+\Delta y}\right] \\
& +\Delta x \Delta y\left[\left.\left(\rho v_{z}\right)\right|_{z}-\left.\left(\rho v_{z}\right)\right|_{z+\Delta z}\right]
\end{aligned}
$$

Here the symbol $\rho$ is the fluid density in $\Delta V$. Separating each side of the matching by $\Delta V$, carrying the $\lim _{\Delta v \rightarrow 0}$, and citing the explanation of the partial imitative direct to:

$$
\frac{\partial \rho}{\partial t}=-\left[\frac{\partial}{\partial x}\left(\rho v_{x}\right)+\frac{\partial}{\partial y}\left(\rho v_{y}\right)+\frac{\partial}{\partial z}\left(\rho v_{z}\right)\right]
$$

This can be uttered in a concise manner as:

$$
\frac{\partial \rho}{\partial t}=-\underline{\nabla} \cdot(\rho \underline{V})
$$

Here, $\underline{V}$ is the rate vector and $\underline{\nabla}$ is the 'inclination' of mechanism. In rectangular combinations, it is noteworthy here that:

$$
\underline{\nabla}=\hat{\imath} \frac{\partial}{\partial x}+\hat{\jmath} \frac{\partial}{\partial y}+\hat{k} \frac{\partial}{\partial z}
$$

Statement $\frac{\partial \rho}{\partial t}=-\underline{\nabla} \cdot(\rho \underline{V})$ is the changing form of the continuity equation. A reshuffle of any statement directs to the corresponding term:

$$
\frac{D \rho}{D t}=-\rho(\underline{\nabla} \cdot \underline{V})
$$

This time, the considerable derivation $\frac{D}{D t}$ is labeled as:

$$
\frac{D}{D t}=\frac{\partial}{\partial t}+\underline{V} \cdot \underline{\nabla}
$$

A great number of fluids bumped into a polymer dispensation in functions are fundamentally incompressible that relates to the fluid density in a constant way. Thus, the simple $\rho$ is a meaning of time or space. Besides, the continuity equation decreases to:

$$
\underline{\nabla} \cdot \underline{V}=0
$$

The measurement of the continuity can be obtained in a same manner-in any suitable organizing methods. Its growths in the three possibly used coordinating systems (spherical, cylindrical and rectangular) are given below:

Rectangular coordinates:

$$
\frac{\partial \rho}{\partial t}+\frac{\partial}{\partial x}\left(\rho v_{x}\right)+\frac{\partial}{\partial y}\left(\rho v_{y}\right)+\frac{\partial}{\partial z}\left(\rho v_{z}\right)=0
$$

Cylindrical coordinates:

$$
\frac{\partial \rho}{\partial t}+\frac{1}{r} \frac{\partial}{\partial r}\left(r \rho v_{r}\right)+\frac{1}{r} \frac{\partial}{\partial \theta}\left(\rho v_{\theta}\right)+\frac{\partial}{\partial z}\left(\rho v_{z}\right)=0
$$

Spherical coordinates:

$$
\begin{gathered}
\frac{\partial \rho}{\partial t}+\frac{1}{r^{2}} \frac{\partial}{\partial r}\left(\rho r^{2} v_{r}\right)+\frac{1}{r \sin \theta} \frac{\partial}{\partial \theta}\left(\rho \sin \theta v_{\theta}\right) \\
+\frac{1}{r \sin \theta} \frac{\partial}{\partial \phi}\left(\rho v_{\phi}\right)=0
\end{gathered}
$$

For an incompressible flow density is a constant. Accordingly we have,

Rectangular coordinates:

Cylindrical coordinates:

$$
\frac{\partial v_{x}}{\partial x}+\frac{\partial v_{y}}{\partial y}+\frac{\partial v_{z}}{\partial z}=0
$$

$$
\frac{1}{r} \frac{\partial}{\partial r}\left(r v_{r}\right)+\frac{1}{r} \frac{\partial}{\partial \theta}\left(v_{\theta}\right)+\frac{\partial}{\partial z}\left(v_{z}\right)=0
$$

Spherical coordinates:

$$
\frac{1}{r^{2}} \frac{\partial}{\partial r}\left(r^{2} v_{r}\right)+\frac{1}{r \sin \theta} \frac{\partial}{\partial \theta}\left(\sin \theta v_{\theta}\right)+\frac{1}{r \sin \theta} \frac{\partial}{\partial \phi}\left(v_{\phi}\right)=0
$$

\section{APPLICATION OF CONTINUITY EQUATION}

The common applications of continuity equation are used in pipes, tubes and ducts with flowing fluids or gases, rivers, overall procedure as diaries, power plants, roads, logistics in general, computer networks and semiconductor technologies and some other fields.

\section{A. Continuity equation on tap}

Now, we can have a reflection on a small chunk of running fluid streaming at speed $v$. In some short intervals symbolized by $\Delta t$, this chunk travels a distance that is symbolized as $d=v \Delta t$. The dimensions of fluid have flowed while this point is referred as $V=d A=A v \Delta t$, and $A$ is the crossed-fragmental area. The accumulation for this chunk that relates to the fluid is:

$$
\Delta m=\rho V=\rho A v \Delta t
$$

So, the mass rate of this flow is,

$$
\frac{\Delta m}{\Delta t}=\rho A v
$$

Now, regarding the fluid flow, there creates a steady state which means that the mass is not comprised or reduced. 
Consequently, the extent of mass flow will surely be equal in everywhere. Yet,

$$
\rho A v=\text { const } .
$$

This equation can be written in another way as it is to say that the rate of the mass flow cannot but remain the same as the same fluid invites any of the same point. Thus

$$
\rho_{1} A_{1} v_{1}=\rho_{2} A_{2} v_{2}
$$

Furthermore, the mass cannot but set off elsewhere. Nevertheless, it is normal for a liquid since the uncompressible logic comes into the action. The compactness will surely be the same all over and again. Therefore, $\rho_{1}=\rho_{2}[10]$.

It bears the logic that:

$$
\begin{gathered}
A_{1} v_{1}=A_{2} v_{2} \\
\text { or, } A_{1}^{2} v_{1}^{2}=A_{2}^{2} v_{2}^{2} \\
\text { or, } \quad A_{2}^{2}=\frac{A_{1}^{2} v_{1}^{2}}{v_{2}^{2}} \\
\text { or, } A_{2}^{2}=\frac{\left(A_{1} v_{1}\right)^{2}}{v_{2}^{2}} \\
\text { or, } A_{2}^{2}=\frac{R_{1}^{2}}{v_{1}^{2}+2 g h} \\
\text { or, } A_{2}=\frac{R_{1}}{\sqrt{v_{1}^{2}+2 g h}} v_{2}^{2}=v_{1}^{2}+2 g h \\
\text { or, } A_{2}=\frac{R_{1}}{\sqrt{\left(\frac{R_{1}}{A_{1}}\right)^{2}+2 g h}}
\end{gathered}
$$

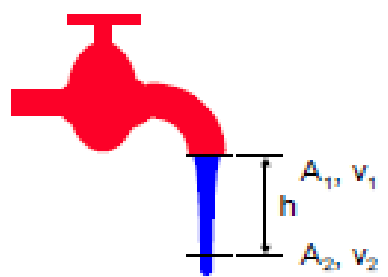

Fig. 2. Decreases in area of a flow water coming from tap

B. Continuity Equation on Flow Rate of a Sluice Gate

For each water depth usage, it determines the flow rate that is symbolized here as $\mathrm{Q}$ and it happens under the sluice gate by using the continuity equation $Q=A_{1} V_{1}=b z_{1} V$. It is to utilize that the Bernoulli as well as continuity equations are to be verified through the theoretical flow rates beneath the sluice gate:

$$
Q=z_{2} b \sqrt{\frac{2 g\left(z_{1}-z_{2}\right)}{1-\left(z_{2} / z_{1}\right)^{2}}}
$$

So far at these culminations, it ought to be assumed that the water profundity centers to the downstream of the gate $\mathrm{z}_{2}$ and hangs about at $61 \%$ of the detachments between the channel base and the bottom point of the gate. Here, it is measured as $\mathrm{z}_{2}=0.61 \mathrm{a}$. The quantifying of the girth, $\mathrm{b}$, of the channel here is $3.3556 \mathrm{~m}$ [11]-[12].

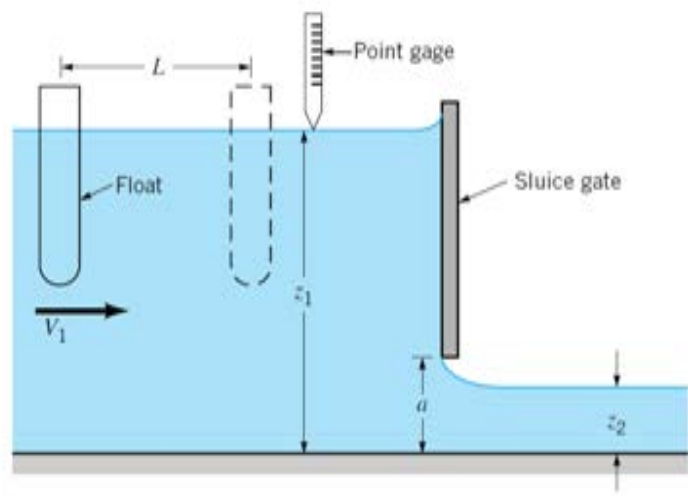

Fig. 3. Sluice Gate Flow rate

TABLE 1: THEORETICAL FLOW-RATE AND EXPERIMENTAL FLOW-RATE

\begin{tabular}{cccc}
\hline \hline $\begin{array}{c}Z_{1} \\
(m)\end{array}$ & $\begin{array}{c}Z_{2} \\
(m)\end{array}$ & $\begin{array}{c}\text { Theoretical flow-rate } \\
Q=z_{2} b \sqrt{\frac{2 g\left(z_{1}-z_{2}\right)}{1-\left(Z_{2} / z_{1}\right)^{2}}}\end{array}$ & $\begin{array}{c}\text { Experimental } \\
\text { flow-rate } \\
Q\left(\mathrm{~m}^{3} / \mathrm{s}\right)\end{array}$ \\
\hline 1.8796 & 0.3098 & 6.1986 & 6.0225 \\
1.9558 & 0.2867 & 5.5654 & 5.4347 \\
2.0066 & 0.2696 & 5.3296 & 5.2274 \\
2.0574 & 0.2541 & 5.1110 & 5.0349 \\
2.1590 & 0.2216 & 4.6089 & 4.5344 \\
2.2098 & 0.2076 & 4.3856 & 4.3252 \\
2.2606 & 0.1889 & 4.0554 & 3.9794 \\
\hline \hline
\end{tabular}

\section{RESUlTS AND DisCUSSION}

We may observe that from Fig.2 the area of water coming from tap is decreasing with respect to height of the tap. So height can be playing a vital role for decreasing area of water coming from tap. The Fig. 4 can be good agreement with previous concept.

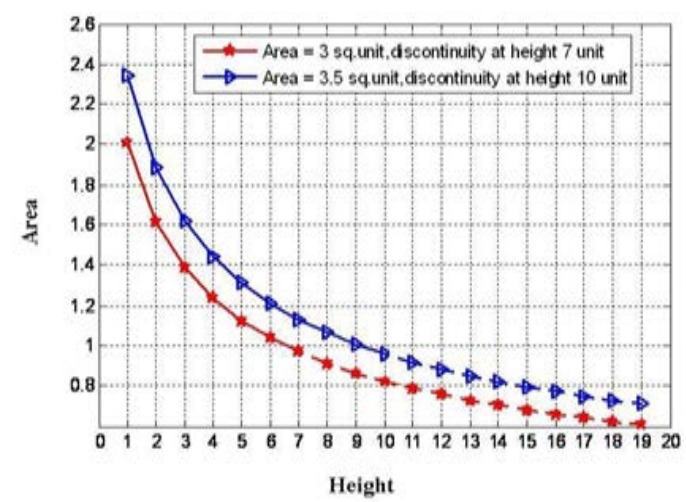

Fig.4. Compare the graph of different area of a flow water coming from tap

By planning and scrutinizing the flow-rate, the ' $Q$ ' is used here as the ordination of the water depth and upstream of the gate as in the abscissas above. 


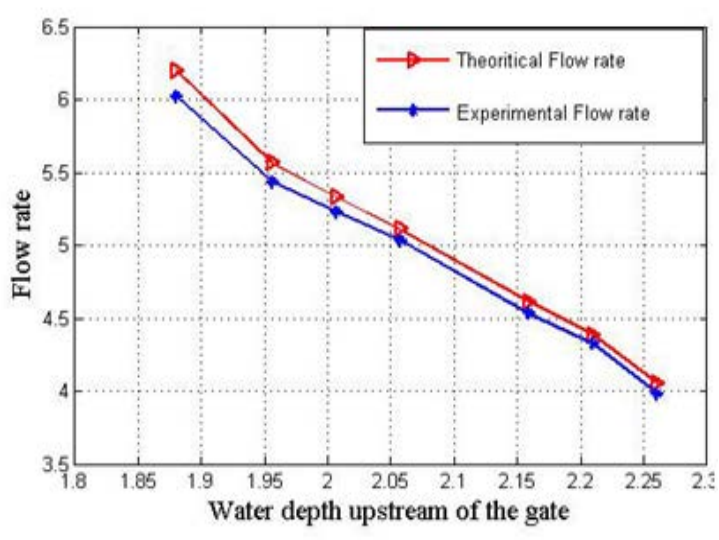

Fig. 5. Compare the graph of theoretical flow-rate and experimental flow-rate

This Figure 5 can be describing the comparison between theoretical flow-rate and experimental flow-rate of the fluid. On the graph, it is seen to be good agreement of theoretical flow-rate and experimental flow-rate as a function of water depth upstream of the gate under the sluice gate by using the aspects of continuity equation.

\section{CONCLUSION}

From the above discussion, we may draw a conclusion that the different continuities have been exposed here for the different areas of taps at different heights. Also, we have experimented to be in the logical agreements of theoretical flow-rates and experimental flow-rate as a core function of water depths for an upstream of the gate under the sluice gate even after having the aspects of continuity equations. Hence, we can approach to this decision that this theoretical procedure will be the most useful one for the fundamental implementations of sluice gate mechanisms. However, I do hope that this idea will open a new window in our scientific minds to go with the modern world as to bring in the welfare of human creativity successfully.

\section{REFERENCES}

[1] Geetam Tiwari etal., "Continuity equation validation for non homogeneous traffic”, Journal of Transportation Engineering, vol. 134, no. 3, pp. 118-127, 2008.
[2] Nyambuya, G. G. , “Avoiding Negative Probabilities in Quantum Mechanics”, arXiv preprint arXiv:1308.4701, 2013

[3] Arbab I, Hisham. M. Widatallah, "Generalized Continuity Equation”, Chin. Phys. Lett. Vol. 27, no. 8, pp. 084703. 2010.

[4] Mohammad Mashud, "Numerical investigation of fluid flow inside a diverging duct”, International Journal of Engineering and Applied Sciences (IJEAS), Vol. 2, no. 3, pp. 63-70, 2010.

[5] Khan, M. N. H. et al., "Leakage Current Paths in PV TransformerLess Single-Phase Inverter Topology and Its Mitigation through PWM for Switching”, International Journal of Power Electronics and Drive Systems, vol. 6, no. 1, pp. 148-159,. 2015

[6] Khan, M. N. H., Khan et al., "Wave shaping with reduced leakage current in transformer-less inverter", International Conference on In Smart Instrumentation, Measurement and Applications (ICSIMA) pp. 1-5 November, 2013.

[7] Khan, M. N. H., Khan et al., "DC-AC inverter with perspective of common mode and wave-shaping”, International Conference on In Smart Instrumentation, Measurement and Applications (ICSIMA) pp. 1-5 November, 2013.

[8] Khan, N. H., "Performance Analysis of Common Issues In the PV Transformer-Less Inverter Topology", European Journal of Engineering Research and Science, vol. 1, no. 1, pp. 43-49, 2016.

[9] Lecture Notes, "Transport Phenomena in Materials Engineering”, MIT Open Course Ware, Massachusetts Institute of Technology, http://www.polymerprocessing.com/notes/root03a.pdf (cited on 10/11/13)

[10] Cutnell Johnson, “Continuity Equation”, Lecture- 20. http://rockpile.phys.virginia.edu/arc00/arch27.pdf. (cited on 15/05/2014).

[11] Sluice Gate Flowrate, Department of Mechanical Engineering, Frederick University, Cyprus. (cited on 15/05/2014).

[12] Cheng A.H-D. et al., "Boundary Calculations of Sluice and Spillway Flows”, J. Hydra. Div., ASCE, vol. 107, no. 10, pp. 1163-1178, 1981.

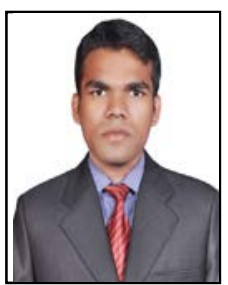

I am Md. Towhiduzzaman. My birth place is Kushtia, Bangladesh. I was born in $1^{\text {st }}$ November, 1986. I completed my B.Sc. (2010) and M.Sc. (2011) degree from Islamic University, Kushtia, Bangladesh in Applied Mathematics and completed my HSC (2004) from Khoksha Degree College, Khoksha. I passed my SSC (2001) from Dansha Secondary School. Presently I have been living at Uttara, Dhaka, Bangladesh for last 2.5 years.

I've been working as a Lecturer in Mathematics, Department of Electrical \& Electronic Engineering, Uttara University, Dhaka, Bangladesh for 2.5 years.

I like to research in mathematical topics especially fluid dynamics. 